\title{
Le réseau international des organismes de bassin
}

\author{
The International Network of Water Basin Authorities
}

\author{
par J. P. Chirouze \\ Agence de l'eau Rhône - Méditerranée - Corse
}

The International Network of Water Authorities was created after the Conference of Rio (June 1992) on the environment and the development. The organization was born in 1994 and 15 countries are participating. The objectives of the network are information exchanges, cooperation in the field of water management, promotion of education... Collective activities are progressively being set up.

\section{I — L'ORIGINE DU RÉSEAU}

Assurer la qualité de la vie sur notre planète, et le développement socio-économique durable de nos sociétés, exige aujourd'hui une gestion rationnelle et équilibrée des ressources en eau.

Les questions soulevées par cette problématique sont complexes. Les réponses doivent notamment permettre :

- de lutter contre les risques d'inondation ou de sécheresse,

- de satisfaire de façon fiable les besoins des populations en eau potable de qualité,

- d'assurer la suffisance alimentaire par l'irrigation appropriée,

- de développer de manière harmonieuse l'industrie, la pratique des loisirs et le tourisme liés à l'eau,

- de combattre les pollutions de toutes origines afin de préserver les écosystèmes aquatiques et, de façon générale, satisfaire les besoins des différents usages.

Ces problèmes ne peuvent plus être abordés de façon sectorielle ou localisée. La recherche de solutions doit, au contraire, associer les Autorités nationales et les Pouvoirs locaux avec les utilisateurs, dans une approche intégrée et respectueuse du milieu naturel, organisée à l'échelle des unités hydrographiques.

Les stratégies, programmes, financements et contrôles doivent être conçus au niveau des bassins versants. Pour les grands fleuves, les mers intérieures ou les mers internationales, des accords de coopération doivent être confortés entre les Pays riverains.

C'est pourquoi, les délégations brésilienne et française, lors de la Conférence des Nations Unies pour l'Environnement et le Développement qui s'est tenue à Rio de Janeiro en juin 1992, ont proposé la création d'un Réseau International des Organismes de Bassin (RIOB).

Les représentants des administrations gouvernementales, chargées de la gestion des ressources en eau, et les organismes de bassin de 15 pays (Brésil, Chili, Côte d'Ivoire, Espagne, France, Hongrie, Indonésie, Maroc, Mexique, Pologne, République Tchèque, Roumanie, Slovaquie, Ukraine et Venezuela) réunis à Aix-les-Bains et à Chambéry, en 1994, à l'invitation des Comités de Bassin et Agences de l'Eau français, ont adopté les résolutions fixant les objectifs et les principes fondateurs du RIOB.

\section{LES OBJECTIFS DU RÉSEAU (voir la Charte d'organisation en annexe)}

Le Réseau s'est fixé pour objectifs :

- de développer les échanges d'informations, d'expériences et d'expertises,

- de promouvoir, dans les programmes de coopération, la gestion de l'eau pour un développement durable,

- de concourir à la réflexion nationale et internationale sur la gestion rationnelle des ressources en eau,

- de faciliter l'élaboration d'outils de gestion institutionnelle et financière,

- de promouvoir des programmes de formation des différents acteurs (élus, usagers, services locaux...),

- d'encourager la formation et l'éducation des jeunes.

\section{III $\square$ LES PARTENAIRES DU RÉSEAU (voir la Charte d'organisation en annexe)}

Le Réseau est ouvert :

- aux organismes gestionnaires de bassins versants nationaux, et le cas échéant, internationaux ;

- aux administrations gouvernementales des pays intéressés par la mise en place d'organisations de bassin ;

- aux organisations de coopération bi ou multilatérale. 


\section{IV — LES PRINCIPES FONDATEURS}

En référence aux recommandations de la Conférence des Nations Unies pour l'Environnement et le Développement de Rio de Janeiro en juin 1992, et de la Conférence Internationale sur l'Eau et l'Environnement de Dublin en janvier 1992 , les partenaires du Réseau doivent appliquer, ou déclarer vouloir appliquer, les principes communs suivants :

- organisation à l'échelle des bassins hydrographiques des modalités d'une gestion intégrée des ressources en eau visant à prévenir les risques naturels dangereux et catastrophiques, à satisfaire de façon rationnelle et équitable les différents usages pour un développement économique durable et à protéger et restaurer les milieux aquatiques :

- mise en place de systèmes de financement des programmes pluriannuels d'aménagement et de protection sur la base du principe " utilisateur-pollueur-payeur ", et de la notion de solidarité de bassin ;

- recherche des modalités d'un partenariat associant à la programmation et à la gestion des organismes de bassin, les autorités nationales et éventuellement les institutions internationales compétentes, les pouvoirs locaux, les utilisateurs de l'eau, les organisations non gouvernementales représentatives concernées et

- développement de capacités d'information des représentants de ces partenaires pour leur permettre d'assumer pleinement les responsabilités et missions qui leur incombent dans le cadre de la politique de bassin.

\section{LA CRÉATION DU RÉSEAU ET SON DÉVELOPPEMENT}

Un Comité de Liaison composé des représentants de 8 pays (France, Brésil, Côte d'Ivoire, Espagne, Indonésie, Mexique, Pologne, Roumanie) a été chargé de définir les modalités de constitution et de fonctionnement du Réseau et d'examiner les candidatures d'adhésion.

Le Secrétariat du Comité de Liaison a été confié à l'Office International de l'Eau, le Gouvernement et les organismes de bassin français ayant accepté d'en prendre en charge les frais de fonctionnement en phase de démarrage.

Au cours des années 1994 et 1995, le bureau de liaison à tenu trois réunions respectivement en Roumanie, en Pologne et au Mexique, qui ont permis de préparer le projet de " déclaration d'adhésion " du RIOB et de " Charte d'organisation et de fonctionnement du Réseau " (cf. annexe).

La première assemblée générale du RIOB s'est tenue à Morelia (Mexique) en mars 1996. Cette Assemblée, dont M. Ernesto Zedillo, Président des Etats Unis du Mexique, a ouvert en personne les travaux, a adopté ces deux textes.

Une journée technique internationale sur " l'information nécessaire à la prise de décision " s'est tenue durant l'Assemblée Générale de Morélia. Ce colloque a permis de réfléchir aux solutions à mettre en œuvre pour développer des réseaux de mesures et d'analyses, optimiser la collecte des données, créer de véritables " observatoires de bassin " synthétisant l'information utile tant pour la planification que pour la fixation de redevances et le suivi des programmes prioritaires d'intervention.

L'Assemblée Générale 1997 du Réseau s'est tenue à Valence (Espagne) en octobre à l'invitation des Autorités espagnoles. Elle a réuni 105 délégués représentant 61 Organismes-Membres de 27 pays.
Les délégués ont décidé que la qualité de membre serait conditionnée au paiement d'une cotisation annuelle fixée à 1000 US\$ pour 1998 destinée à couvrir les frais généraux d'animation du Réseau et par priorité la publication de la "Lettre du RIOB ". Les Agences de l'Eau françaises ont confirmé leur engagement de soutenir financièrement le Secrétariat en 1998 durant la phase de montée en puissance des cotisations.

Une " Journée thématique sur le financement des Organismes de Bassin " a été organisée. A l'issue des présentations d'études de cas et des débats qui les ont suivies, les délégués ont adopté des recommandations correspondant largement au système français :

- établissement de schémas directeurs de gestion des ressources en eau sur 15 à 20 ans,

- élaboration, dans ce cadre, de programmes quinquennaux d'intervention,

- financement reposant principalement sur la tarification des services liés aux usages de l'eau,

- mobilisation de financements complémentaires et création d'une solidarité de bassin par la mise en place de redevances sur les consommations et les pollutions perçues auprès de toutes les catégories d'utilisateurs,

- participation de toutes les catégories d'usagers à la définition de la politique de l'eau dans le bassin,

- utilisation de systèmes modernes d'information sur l'état des ressources en eau et sur les usages.

En juillet 1997 à Brasilia, se sont réunis les dix principaux pays d'Amérique Latine dans lesquels sont en cours des réformes institutionnelles pour le secteur de l'eau. Ils ont créé le Réseau Régional du RIOB pour l'Amérique Latine et adopté à l'unanimité ses statuts. Ils ont également accepté l'invitation des autorités colombiennes pour que la première assemblée générale ordinaire se tienne en Colombie en juin 1998.

\section{VI — LES ACTIVITÉS COLLECTIVES DU RÉSEAU}

Celles-ci commencent progressivement à se mettre en place pour répondre aux objectifs du réseau :

- développer l'échange d'information et d'expérience : “ La Lettre du RIOB ", rédigée par les membres du Réseau euxmêmes, est un lieu privilégié d'échange d'informations sur leurs expériences. Six numéros ont déjà été publiés, dans les trois langues officielles du RIOB (français, espagnol et anglais), dont le dernier a été tiré à plus de 9600 exemplaires diffusés à travers le monde.

Enfin, le serveur Internet " HTTP://WWW.OIEAU.FR/ RIOB/" diffuse depuis novembre 1996, en trois langues (français, anglais, espagnol), toute la documentation publiée dans le cadre du RIOB.

- promouvoir les programmes de coopération : " AQUADOC-INTER " permettra l'échange de documentations institutionnelles à partir de références harmonisées entre les organismes de bassin.

“AQUADOC-INTER " fonctionnera sur Internet et constituera une " bibliothèque virtuelle " rassemblant toute la documentation juridique, administrative et économique sur l'organisation de la gestion de l'eau par bassin dans les pays adhérents au RIOB. 
La première étape de ce projet, qui s'appuie sur des professionnels du traitement documentaire, a été la désignation à titre expérimental de 12 " Centres Documentaires Relais Nationaux " (CDRN) qui seront en fait les " guichets " servant de relais entre les utilisateurs, les bases spécialisées nationales et le système.

Il s'agit de créer les conditions d'échanges au sein d'un réseau en harmonisant les systèmes de références, les modalités de requête et les mises à disposition des copies, donc de rendre transparent pour les utilisateurs l'accès aux informations qu'ils souhaitent, quelle qu'en soit la source.

- concourir à la réflexion internationale : l'Atelier International sur les Schémas Directeurs de Bassin s'est tenu, à l'initiative des Autorités Roumaines, à Constanza (Roumanie) du 31 août au 4 septembre 1996 et a permis à 31 participants de onze pays de faire le point de l'organisation des institutions de l'eau dans les différents pays concernés par le RIOB : il s'est agi d'un véritable cours d'administration comparée, fort utile pour développer les principes, adaptés à chaque situation locale, d'une meilleure gestion des ressources.

- promouvoir les programmes de formation : un cours international trilingue de 2 semaines sur " la création des organismes de bassin " sera aussi organisé conjointement par la France et l'Espagne.

- concourir à la réflexion internationale : plus récemment la Conférence Internationale de Paris sur " I'Eau et le Développement Durable " a été l'occasion pour le RIOB d'organiser un atelier sur la gestion de l'eau par bassin versant, l'intérêt de la planification à long terme, l'obligation d'une forte association des usagers et l'efficacité des systèmes de redevances spécifiques pour le financement des programmes.

La Conférence de Paris a permis de faire largement connaître le RIOB et les possibilités de coopération internationale qu'il permet.

\section{VII $\square$ CONCLUSIONS}

Le RIOB est maintenant constitué autour d'un noyau de membres fondateurs qui ont appris à se connaître et sont en mesure d'échanger leur savoir-faire en matière de gestion globale de la ressource en eau à long terme, ceci en tenant compte très pragmatiquement des contraintes locales géographiques, sociales, économiques et institutionnelles.

Il s'agit d'un réseau informel (le RIOB n'est pas doté de personnalité juridique propre), reposant sur la volonté de travail en commun de ses membres. La France y est très présente : les Agences de l'eau participent activement à la vie du Réseau dont le secrétariat est tenu par L'Office International de l'Eau.

Le réseau peut être utilement consulté, très en amont, sur la préparation de projets structurants dans le domaine de l'eau (irrigation, eau potable, assainissement des villes, hydroélectricité...) notamment pour permettre à ces projets de mieux s'inscrire dans la problématique globale de gestion des ressources en eau et dans le contexte institutionnel local.

Le RIOB est de création récente (1994). L'enjeu porte maintenant sur la possibilité de faire connaître l'originalité et l'utilité du Réseau aux acteurs (autorités nationales ou locales, compagnies d'aménagement, bureaux d'études, entreprises concessionnaires...) qui envisagent des programmes d'actions structurants dans le domaine de l'eau (création ou amélioration d'infrastructures, réorganisation, ...) ou sont consultés pour leur élaboration.
En ce qui concerne la France, nous avons notre place à tenir pour faire connâtre l'expérience acquise, notamment au moment où s'élabore une nouvelle directive cadre de l'Union Européenne.

La participation française au RIOB offre également l'opportunité de consolider une présence à international qui peut concourir à favoriser l'offre d'entreprises françaises sur un marché très concurrentiel.

C'est notamment pour l'ensemble de ces raisons qu'il paraissait intéressant de présenter le RIOB aux membres de la SHF.

\section{ANNEXE \\ RÉSEAU INTERNATIONAL DES ORGANISMES DE BASSIN}

\section{Charte d'organisation et de fonctionnement}

\section{Les objectifs du réseau}

Le Réseau International des Organismes de Bassin a pour objectifs :

- de développer des relations permanentes entre les organismes intéressés par une gestion globale des ressources en eau par grands bassins hydrographiques et favoriser entre eux des échanges d'expériences et d'expertises,

- de promouvoir dans les programmes de coopération les principes et moyens d'une gestion rationnelle de l'eau pour un développement durable,

- de faciliter l'élaboration d'outils de gestion institutionnelle et financière, de programmation, d'organisation des banques de données, de modèles adaptés aux besoins,

- de promouvoir des programmes d'information et de formation des élus locaux, des représentants des usagers et des différents acteurs de la gestion de l'eau ainsi que des dirigeants et des personnels des organisations de bassin membres,

- d'encourager l'éducation des populations et notamment des jeunes,

- d'évaluer les actions engagées et d'en diffuser les résultats.

\section{Les membres du réseau}

Le Réseau est ouvert :

- aux organismes gestionnaires de grands bassins versants nationaux ou fédéraux, et le cas échéant, transfrontaliers, ainsi qu'aux structures de coopération qu'ils ont pu développer entre eux.

Par " Organismes de Bassin ", il faut entendre des établissements publics ayant une existence légale ou en cours de création dans le cadre de la législation en vigueur dans son pays, ayant une personnalité juridique et disposant d'un budget propre.

- à l'administration gouvernementale chargée de l'eau dans chacun des pays appliquant, ou ayant déclaré vouloir appliquer, une gestion globale de l'eau :

- organisée par grandes entités hydrographiques (bassins versants),

- associant les Administrations, les Pouvoirs locaux, ainsi que les usagers des différents secteurs,

- disposant de ressources budgétaires spécifiques obtenues par l'application du principe " utilisateurs-pollueurspayeurs". 
Par grandes entités hydrographiques, il faut comprendre les bassins versants des grands fleuves et des lacs, à l'exclusion de leurs différents petits affluents.

- aux organisations de coopération bi ou multilatérale soutenant des actions de structuration institutionnelle au niveau des grands bassins versants et aquifères.

Les membres acquièrent cette qualité dès lors qu'ils ont fait acte de candidature au Réseau en signant la " Déclaration d'adhésion".

\section{Les observateurs permanents}

Les autres organismes publics intéressés par le Réseau peuvent demander à participer à ses activités en qualité d'observateurs permanents, sous réserve de l'accord de l'Assemblée Générale, sur proposition du Bureau de Liaison.

Ils peuvent assister à l'Assemblée Générale sans voix délibérative.

\section{L'organisation du réseau}

Le Réseau est une structure souple reposant sur la volonté de travail en commun de ses membres.

Il n'est pas doté d'une personnalité juridique propre, ni d'un statut d'organisation internationale, mais il est simplement régi par la présente " Charte d'organisation et de fonctionnement".

\section{Président du Réseau}

C'est le président de l'Assemblée Générale.

Il est désigné par le ou les membres-hôtes qui invitent pour la réunion.

Il représente le Réseau jusqu'à la réunion suivante de l'Assemblée Générale.

Il s'assure de I'application des recommandations du Bureau et des conclusions de l'Assemblée Générale jusqu'à la prochaine réunion et organise le travail du Secrétariat.

\section{Bureau de liaison}

Il est composé du Président en exercice du Réseau et des deux précédents Présidents, ainsi que :

. 1 membre pour l'Afrique,

. 2 membres pour l'Amérique Latine,

. 1 membre pour l'Asie,

. 2 membres pour l'Europe Centrale et Orientale,

. 2 membres pour Europe Occidentale.

Sa composition peut être revue à chaque Assemblée Générale en fonction des adhésions à venir pour chaque région géographique.

Les membres de chaque région géographique, ci-dessus, proposent à l'Assemblée Générale, par consensus entre eux, le ou les pays qui les représentent au Bureau de Liaison. Les membres de chacun de ces pays retenus désignent ensuite entre eux la personnalité qui siège au Bureau.

Le Bureau tient de 2 à 3 réunions par période de 12 mois entre deux Assemblées Générales.

Il est présidé par le représentant désigné par le ou les membres-hôtes de la réunion.

Il prépare la prochaine réunion de l'Assemblée Générale.

Il exécute les conclusions de l'Assemblée Générale précédente.

Il coordonne les projets communs.
Il analyse les candidatures des observateurs permanents ainsi que des nouveaux membres du Réseau dont il propose l'adhésion à la prochaine Assemblée Générale.

Il adopte ses recommandations par consensus entre ses membres présents.

Les personnalités siégeant au Bureau peuvent se faire remplacer en cas d'empêchement pour se rendre à une des réunions.

Le ou les membres-hôtes de la prochaine réunion du Bureau en fixe la date après s'être assuré que les $2 / 3$ de ses membres au moins pourront y être présents.

\section{Assemblée générale}

Elle réunit l'ensemble des membres du Réseau.

L'Assemblée Générale est présidée par le président du Réseau.

Elle tient une réunion ordinaire chaque année et peut tenir des réunions extraordinaires.

Les Assemblées Générales Ordinaires se tiennent chaque année autant que possible dans une région géographique différente.

\section{L'Assemblée Générale :}

- approuve le texte de la "Déclaration d'adhésion" et de la " Charte d'organisation et de fonctionnement " ainsi que leurs modifications éventuelles,

- officialise l'adhésion de nouveaux membres sur proposition du Bureau de Liaison ainsi que les demandes de participation des observateurs permanents,

- oriente les activités du "Réseau ",

- approuve les projets communs proposés par le Bureau de Liaison, et organise si nécessaire les " cellules opérationnelles " chargées de leur mise en œuvre,

- assure la promotion du "Réseau " auprès des Autorités Nationales et des Organisations de Coopération bi et multilatérales concernées,

- définit la composition du Bureau de Liaison.

Les conclusions de l'Assemblée Générale sont obtenues par consensus entre les Membres présents. A défaut d'accord consensuel, le Président pourra, en dernier recours, procéder à un vote pour obtenir une majorité qualifiée des deux tiers des membres présents.

Des personnalités qualifiées ou des organismes intéressés peuvent être invités par le ou les membres-hôtes à assister à l'Assemblée Générale sans prendre part à ses délibérations, au même titre que les observateurs permanents.

\section{$\underline{\text { Secrétariat }}$}

Le Secrétariat du Réseau est assuré par :

- un Secrétariat de Présidence, organisé par le ou les organismes-hôtes qui assurent l'organisation des réunions du Bureau de Liaison et de l'Assemblée Générale,

- un Secrétariat Technique Permanent désigné, par période de quatre ans, par l'Assemblée Générale sur proposition du Bureau de Liaison, parmi les candidatures présentées.

Il est chargé de la préparation des dossiers des Bureaux de Liaison et des Assemblées Générales, et de la rédaction des comptes-rendus sous l'autorité du Président, de l'édition périodique de " La Lettre du Réseau ", de l'animation du Réseau et du suivi des projets communs, en liaison étroite, le cas échéant, avec les " cellules opérationnelles correspondantes". 
Le Responsable du Secrétariat Technique Permanent participe aux réunions de l'Assemblée Générale et du Bureau de Liaison.

Le Responsable de l'organisme qui assurait le Secrétariat Technique Permanent durant la période précédente est également invité à ces réunions durant les quatre années suivantes afin d'assurer la continuité des actions entreprises et d'apporter sa collaboration au Secrétariat en titre.

\section{Adresse postale du réseau}

C'est celle du Secrétariat Technique Permanent.

\section{Réseaux régionaux}

Des Réseaux Régionaux peuvent être constitués à l'initiative des Organismes membres d'une même région géographique, après accord de l'Assemblée Générale, sur proposition du Bureau de Liaison.

Le Président du Réseau et le Représentant du Secrétariat Technique Permanent sont invités aux réunions et manifestations organisées dans le cadre de chaque Réseau Régional.

\section{Les principes de financement}

Les activités du Réseau sont financées de la façon suivante :

Réunions du Bureau de Liaison et de l'Assemblée Générale :

- voyages à la charge des membres participants,

- frais de séjour, logistique, visites techniques, traduction et Secrétariat de Présidence à la charge du ou des Membres hôtes dans la limite d'un représentant officiel par Organismemembre du Réseau.

Projets communs :

moyens de réalisation à négocier par le Bureau de Liaison auprès des bailleurs de fonds bi et multilatéraux, les gouvernements et organismes intéressés, avec l'appui d'une cellule opérationnelle, si nécessaire, et du Secrétariat Technique Permanent.

Cotisation annuelle :

Le principe et le montant d'une cotisation annuelle versée par les Organismes-membres seront envisagés lors de la deuxième Assemblée Générale du Réseau. 